\title{
Téoros
}

Revue de recherche en tourisme

\section{Les loisirs de proximité : nouvelles tendances}

\section{Yves Raynouard}

Volume 8, numéro 1, mars 1989

France-Québec

URI : https://id.erudit.org/iderudit/1080354ar

DOI : https://doi.org/10.7202/1080354ar

Aller au sommaire du numéro

Éditeur(s)

Université du Québec à Montréal

ISSN

0712-8657 (imprimé)

1923-2705 (numérique)

Découvrir la revue

Citer cet article

Raynouard, Y. (1989). Les loisirs de proximité : nouvelles tendances. Téoros, 8(1), 22-24. https://doi.org/10.7202/1080354ar d'utilisation que vous pouvez consulter en ligne.

https://apropos.erudit.org/fr/usagers/politique-dutilisation/ 


\section{Les loisirs de proximité: nouvelles tendances}

Évoquer un changement profond de la société française dans le domaine du "temps libéré sur le temps de travail", dans un numéro de la revue destiné entre autres à comparer les situations au Québec et en France, n'est pas chose aisée. Certes, il y a entre nos deux pays de nombreux points communs. Nous sommes engagés, les uns comme les autres, dans un processus historique nouveau de baisse du volume global de travail. On redistribue alors du temps. Mais si, en France, cela se porte sur une cinquième semaine de congés, au Québec comme en Amérique du Nord, l'affectation en temps de loisirs se fait de manière plutôt "émiettée" depuis longtemps.

L'une des mutations que nous connaissons en France - l'apparition des pratiques séquentielles de loisirs corrélativement à la diminution du phénomène des grandes vacances - tendrait donc à nous rapprocher de nos cousins d'Amérique.
Par contre, on peut s'interroger sur la notion de proximité et ce qu'elle signifie dans les deux cas, surtout avec des tissus urbains aussi differrents. La proximité dans l'espace en France, c'est, pour une sortie de la journée, un rayon inférieur à $100 \mathrm{~km}$ et, pour un week-end, environ $250 \mathrm{~km}$, alors qu'au Québec rares sont les sorties a la journee et pour le week-end on peut au moins doubler la distance parcourue par le Français...

Mais, là Egalement, on observe une tendance à la ressemblance dans les comportements. avec l'apparition de "l'espace européen" . A l'heure du TGV et des vols charters, la plupart des villes européennes deviennent proches dans le temps. La différence avec le Québec est que les échanges "interprovinciaux" que nous connaissons ne sont pas encore répandus chez cux (alors que les courts séjours chez leurs voisins américains, eux, le sont).

Ce préambule étant fait, nous allons décrire ce qui nous parait representer aujourd'hui les principales tendances qui marquent un changement profond de la société française en matière de loisirs.

\section{Les tendances au changement}

Le loisir, objet d'une conquête sociale en France en 1936, va s'appeler dans les années 50 et 60 "vacances et grandes vacances" . Cela correspond à la fois à l'accession à un modele de consommation, à un moyen de se liberrer des contraintes du travail et à une période de reconstitution de son énergie (pour ne pas dire de sa force de travail...). C'est aussi la conquête de nouveaux espaces dans une situation très souvent de fuite de la ville et de rupture avec la vie quotidienne. En période de forte expansion, le progrès social qui comprend notamment "l'accès du plus grand nombre aux vacances" n'est pas mis en doute.

La crise est arrivée et la France a été le dernier pays occidental à se mettre au régime de l'austérité et de la rigueur. La France a done cru au cours des années 70 qu'elle allait sortir indemne, notamment dans le domaine du tourisme, de cette situation de crise. Pendant cette période, le volume annucl de travail professionnel en France a été réduit de $10 \%$. On a alors commencé à parler de temps libre et de cinquième semaine de congés payés. En 1977, les Français, quelle que soit leur catégorie de revenus, optaient "pour davantage de temps libre plutô que davan- tage de salaire" "Mais "temps libre" voulait encore dire "vacances" en 1981 et l'offre professionnelle était "multi-propriétéé", "villages de vacances" et "sites exotiques". .. Et puis, au début des années 80 , les indicateurs concernant les vacances ont commencé à stagner. L'ère de la rigueur est arrivée avec, comme conséquence majeure, la stagnation du pouvoir d'achat de nombreuses catégories de populations. Les trois-quarts des Français actifs ont alors dit: "On prefere davantage d'argent que de temps libre".

Et lorsqu'ils sont interrogés sur ce qu'ils veulent faire de leur temps de vacances supplémentaire (la sème semaine de congés à été, rappelons-le, généralisće en 1981), ils parlent de "week-ends prolongés", de "ponts agrandis" ou de "courts séjours" en général. Ce qui vient en dernier, ce sont des jours de vacances en plus.

Ainsi, ce qui apparait aujourd'hui dans les enquétes qui sont faites sur les attentes concernant les affectation du temps libéré par le travail professionnel, c'est une affectation "en miettes" d'un certain nombre de temps de loisirs. Mais pour quoi faire?

La première tendance forte est celle de la recherche de nouvelles sociabilites. On souhaite multiplier les rencontres et les relations sociales spontanćes ou volontaires de toutes sortes (on veut rompre en particulier l'isolement vécu de plus en plus souvent dans la ville).

L'INSEE a réalisé à 20 ans d'intervale (67-87) deux enquêtes sur les pratiques de loisirs des Français. Ceux-ci marquent un intérêt plus vif qu'il y a 20 ans pour les réunions, les associations et les rencontres. Après l'emprise croissante de la télévision sur la vie quotidienne, il s'agit du trait le plus marquant de l'evolution des loisirs entre 1967 et 1987 .

La seconde tendance est celle de ce que l'on pourrait appeler "la recherche de viralite et de sante". C'est la prise de conscience du corps, de la prévention qu'il requiert et du plaisir que l'on peut en tirer, des lors qu'il retrouve un certain dynamisme. Dans ses travaux sur les "sociostyles", le Centre de Communication $A$ vancée ( $C C A)$, relève plusieurs comportements dominants, notamment "la mise au vert" et la "remise en forme" qui vont souvent de pair. Les plus grands consommateurs seraient les "recentrés matérialistes" ", autrement dit les classes moyen- 
nes ( $26 \%$ de la population totale). On peut noter que de "jeunes retraités" sont de plus en plus sensibles à cette recherche de bienêtre au moyen d'activités physiques et corporelles. De ce point de vue, le Québec, avec la pratique du golf, a largement devancé la France en répondant depuis un certain temps déjà à cette demande.

La troisième tendance, peu de personnes en parlent à ce jour. Il s'agit des loisirs à partir de l'entreprise. En France, nous sommes dotés depuis la fin de la dernière guerre, d'une institution qui peut intervenir notamment sur les loisirs: le Comité d'Entreprise (CE). Jusqu'à présent les activités proposées par cette institution gérée le plus souvent par des représentants d'organisations syndicales, étaieṇt plutôt perçues par beaucoup de salariés comme étant astreignantes. Face au déterminisme individualiste (ou de recherche d'autonomie) des salariés pour leurs loisirs, les propositions des CE apparaissaient plutôt comme des formules "collectivistes" même si certains d'entre cux les acceptaient par nécessité financière et même si nombre de CE s'ouvraient aux besoins des communes d'accueil.

Aujourd'hui, où l'on parle de plus en plus souvent en France de "projet et d'esprit d'entreprise" avec des directions souhaitant que les gens vivent leur entreprise comme autrefois l'on vivait son village, il est probable que la cloison entre le vécu du loisir et le vécu du temps travaillé va s'effacer en partie. Tout dépend de la renaissance ou non des relations de voisinage autour du quartier. Si celles-ci continuent à se détériorer, l'entreprise dans laquelle on travaille sera de plus en plus vécue comme une des cellules d'appartenance, autrement dit comme un élément primordial du nouveau tissu social. Un indicateur intéressant vis-à-vis de cette tendance est représenté par le développement des voyages de stimulation (incentives). En forte progression aux États-Unis, cette manière de méler étroitement loisir et travail, va-t-elle connaître le même succès en France? Nous le pensons mais bien entendu le débat reste ouvert sur cette question. Il faut d'ailleurs noter que l'un des moyens de rapprocher ou d'ouvrir l'entreprise sur le quartier est représenté par le développement des services de proximité dont ceux concernant les loisirs, le sport, la culture... Affaire à suivre....

\section{Les équipements à investissements lourds}

A la fin des années 60 et au cours des années 70 , pour les Français qui avaient les moyens de "tsortir de la ville" pendant leurs congés courts $(2,3,4$ jours), il y avait grosso modo trois solutions: la résidence secondaire, les sports d'hiver ou un site "ensoleille" (en France ou à l'étranger) ct le Parc ou la Base de loisirs. En ce qui concerne cette dernière solution, il faut signaler qu'il s'agissait au départ, pour le Pare, d'un lieu d'hébergement "sec" (maison mobile ou caravane) et pour la Base, d'une pratique de "loisirs au quotidien',

TABLEAU 1

Évolution de quelques pratiques de "loisirs au quotidien" entre 1967 et 1988

\begin{tabular}{|c|c|c|}
\hline Proportion des individus ayant partiqué l'activité & 1967 & $1987-1988$ \\
\hline Regarder la télévision tous les jours ou presque & 51 & 82 \\
\hline Lire un quotidien tous les jours ou presque & 60 & 42 \\
\hline Lire une revue ou un magazine régulièrement & 56 & 79 \\
\hline Lire au moins un livre par mois & 32 & 31 \\
\hline Aller au cinéma au moins une fois par mois & 18 & 18 \\
\hline Aller au cinéma chaque semaine ou presque & 6 & 4 \\
\hline Aller au theitre au moins une fois par an & 21 & 18 \\
\hline Assister à un spectacle sportif uu moins 5 fois par an & 17 & 9 \\
\hline Avoir visité un salon ou une foire exposition depuis un an & 33 & 56 \\
\hline Avoir visité un muséc depuis un an & 18 & 32 \\
\hline Avoir visité un chäteau ou un monument depuis un an & 30 & 41 \\
\hline Sortir le soir au moins une fois par mois & 30 & 48 \\
\hline Aller au café au moins une fois par semaine & 24 & 17 \\
\hline Aller au restaurant au moins une fois par mois & 8 & 25 \\
\hline Recevoir des parents ou amis pour un repas au moins une fois par mois & 37 & 61 \\
\hline Participe régulièrement à au moins une association & 11 & 18 \\
\hline Réparer une voiture de temps en temps et "avec plaisir" & 10 & 12 \\
\hline Faire de la couture ou du tricot de temps en temps et "avec plaisir" & 28 & 38 \\
\hline Jardiner tous les jours ou presque à la belle saison & 20 & 19 \\
\hline Ecouter la radio tous les jours ou presque & 67 & 75 \\
\hline Danser au moins 5 ou 6 fois par an & 20 & 30 \\
\hline Jouer aux cartes ou à d'autres jeux de sociétés chaque semaine ou presque & 13 & 18 \\
\hline Jouer de la musique régulièrement ou parfois & 4 & 7 \\
\hline Faire une colection & 16 & 22 \\
\hline
\end{tabular}

Sources: enquêtes INSEE 1967 et 1987

TABLEAU 2

Quelques pratiques de loisirs en 1988

\begin{tabular}{|l|c|}
\hline Proportion des individus ayant partiqué l'activité suivante... & En \% \\
\hline Partir en vacances (4 nuits au moins) chaque annće ou presqué & 54 \\
Partir en court déplacement (1 à 3 nuits) au moins 3 fois par an & 30 \\
Chasser au moins une fois dans la saison & 5 \\
Pécher au moins une fois dans l'annće & 18 \\
Marcher à pied régulièrement en ville au moins une heure & 28 \\
Marcher à pied régulièrement dans la nature au moins une heure & 31 \\
Faire de temps en temps unè randonnée d'au moins une journée à pied ou à vélo & 12 \\
Faire de temps en temps des pronenades d'environ une demi-journée à vélo & 11 \\
Jouer de temps en temps à la pétanque ou autre jeu de boules (bowling non compris) & 34 \\
Faire de la gymnastique, du yoga, de là danse ou un autre exercice physique & \\
d'entretien (en dehors de l'éducation physique obligatoire à l'éole) & 15 \\
Pratiquer régulièrement un sport d'équipe & 5 \\
Bricoler tous les jours ou presqué & 14 \\
Faire de la peinture, de la sculpture ou du dessin & 11 \\
Faire régulièrement des mots croisés & 20 \\
Jouer de temps cn temps au tiercé ou à d'autres courses de chevaux & 13 \\
Jouer de l'argent à un jeu comme le loto ou la loterie nationale & 37 \\
\hline
\end{tabular}

Source: enquête INSEE - mai 1978 à mai 1988

La France est ainsi arrivếe, au début des années 80 , à être dotée non seulement d'un parc de résidences secondaires important (2 260 000) mais aussi de plusieurs centaines de Bases et de Pares de loisirs. Mais étaitce suffisant pour absorber la progression des séjours courts des Français? (dans l'enquête de l'INSEE réalisće de mai 1987 à mai 1988 , environ un tiers des 10872 personnes qui ont répondu, sont parties en court déplacement - 1 à 3 nuits - au moins 3 fois dans l'année).

Une offre nouvelle va apparaître avec l'arrivée d'acteurs-investisseurs (groupes bancai- res,investisseurs institutionnels, entreprises de BTP,...) estimant a priori rentables des équipements spécifiques correspondant à ce que l'on pourrait appeler "les loisirs prêts à consommer".

Ces equipements vont constituer des investissements lourds avec une durabilité relativement réduite. $\AA$ part les parcs aquatiques qui vont être des créations françaises (méme s'il en existe déjà à l'étranger lorsque le premier se réalise à Cap d'Agde), les autres équipements vont représenter des "transferts d'idées" déjà mises en oeuvre à l'étranger. 
$\mathrm{C}^{4}$ est le cas en particulier des parcs à thème "copiés" sur ceux des Américains, des Allemands ou des Hollandais. Car même si les themes d'Asterix ou de Schtroumpf sont Français, l'idée du parc d'attractions dans lequel vous trouvez aussi des commerces, des restaurants et même des hôtels, ne vient pas de notre hexagone.

Mais là où la copie pose le plus de questions vis-à-vis du comportement des clientèles françaises, c'est le Parc Résidentiel de Loisirs (PRL). La France est avant tout, rappelons-le, un pays touristique (en tant que lieu de destination et en tant que pays à fort pourcentage de tourisme domestique ou national). La nouvelle "copie" c'est le PRL, type "Center Park". Onze parcs résidentiels ont déjà êté réalisés en Hollande, Belgique, Angleterre, Allemagne, La France vient $\mathrm{d}^{*}$ accueillir le $12 \mathrm{eme}$. L'objectif est de faire fonctionner toute l'année un centre comprenant plusieurs centaines de bungalows avec une bulle tropicale et des équipements sportifs très diversifiès, le tout implanté dans un vaste espace naturel réservé. Les clienteles sont supposées être en majorité des revenus supérieurs et les tranches hautes des revenus intermédiaires habitant les grandes agglomérations proches. Pour celui qui vient d'ouvrir en Normandie, outre les etrangers des pays limitrophes du notd de l'Europe, on compte beaucoup sur la clientèle de l'île-de-France.

Le Club Méditerranée se lance aussi dans le genre Center Park, avec une première installation dans l'Yonne. Le ciblage des clientèles est cependant sensiblement différent. Pour esperer faire tourner les installations tout au long de l'année, le Club Med va tenter de faire venir, simultanément ou non, différentes clientèles: des étrangers pendant l'été, des populations urbaines pour des courts sejours et des stages de formation ou des séminaires d'affaires.

On voit l'importance que peuvent prendre les tendances au changement que nous avons précédemment énoncées. S'il se confirme que l'entreprise dans laquelle on travaille constitue de plus en plus une cellule d'appartenanoe à partir de laquelle se développent relations et loisirs, il est probable que ces "mondes clos" que constituent les Center Parks connaltront un grand succès. Par contre, si les relations de voisinage autour du logement et du quartier de ville renaissent, beaucoup de personnes rechercheront à vivre un temps de loisirs en rupture avec le temps de travail. Ce sera alors, dans la ville ou en périphérie de la ville, que les tendances "à de nouvelles sociabilites" ou "d une recherche de vitalite et de sante" pourront trouver une réponse.

Mais aujourd'hui, si l'offre est naissante en matiere d'equipements "pour des loisirs prèts à consommer", elle est quasi inexistante, en France, sur le plan des "services pour des loisirs de proximite"
Notons que ces deux voies de création et d'exploitation d'équipements, si elles se situent dans "une economie des loisirs", représentent des finalités différentes: rentabilité Économique comparable à d'autres secteurs industriels dans le premier cas, rentabilité à la fois économique et sociale dans le second. Ce qui veut dire pour les collectivités territoriales une implication financière plus ou moins importante selon qu'il s'agit de permettre la réalisation d'un équipement pour populations solvables avec une exploitation dégageant de bons résultats, ou bien un service d'intérêt collectif ouvert à l'ensemble de la population d'un quartier.

\section{Les équipements et services}

Imaginons quelle est l'offre qui va pouvoir se mettre en place dans ou a proximité des villes.

Sur la base des deux premières tendances au changement énoncées ci-avant, la démarche va probablement plus tenir compte de l'importance croissante des improductifs et des inactifs (enfants, femmes, chômeurs, retraités).

Il n'est pas question ici de dresser la liste de tout ce qui pourrait etre mis en oeuvre pour répondre aux pratiques des loisirs hors du domicile.

Présentons quelques exemples. Dans l'optique de la prevention corporelle et de la recherche de bien-être physique, on peut imaginer un centre de remise en forme au niveau d'un quartier. Celui-ci comprendrait, outre un bassin réservé à l'apprentissage de la natation, hammam ou sauna, salle de gymnastique et divers locaux de soins esthétiques ou de massage. Toutes les catégories d'âges seraient concernées par cette installation. Certaines pourraient l'utiliser de manière exclusive à certaines heures: personnes agées pratiquant de la gymnastique volontaire ou bien enfants apprenant la natation pendant que leurs parents font des courses. Le plein emploi de l'équipement devrait permettre d'offrir des tarifs abordables par toutes les bourses (de l'ordre du prix d"une place de cinema pour deux heures).

Autre equipement favorisant les rencontres: un espace "multi-jeux de sociéte" . L'offre et la demande en matière de jeux sont extrềmement diversifiées. Par exemple, si les jeux de simulation sont plutôt pratiqués par les 18 a 25 ans, parmi les jeux de l'esprit on distinguera notamment une moyenne d'âge superieure à 45 ans pour le bridge, inférieure a 35 ans pour le Go et toutes les catégories d'äge concernées par le scrabble...

Le concept des produits, mis au point par CODATEL - JADES - peut paraitre ambitieux puisqu'il vise à réunir en un mểme lieu mais pas nécessairement dans le même temps, différentes catégories de clientèles pratiquant une grande variété de jeux: c'est la formule des "jeux à la carte" ". Cette formule peut aussi s'imaginer pendant un séjour de vacances ou de loisirs de courte durée.
La demande est multiforme et comme la plupart des types de jeux varie en fonction des catégories d'âge, l'aménagement de l'espace et les equipements doivent permettre à la fois, la venue simultanée de différentes catégories de clientèles et leur accueil tout au long de l'année. Il faut donc que JADES puisse être ouvert de manière souple à des individuels comme à des membres d'associations ou de clubs, à des compétiteurs comme à des non-inities, à des jeunes ou à des moins jeunes. L'un des meilleurs moyens d'utiliser JADES toute l'année, réside notamment dans sa conception d'outil de formation pour le personnel des entreprises ou encore pour des classes d'enfants (jeux pédagogiques).

En guise de conclusion: peut-on faire "cohabiter" les loisirs urbains et péri-urbains avec le tourisme urbain?

Loisirs urbains et peri-urbains sont avant tout une réponse aux besoins des populations locales. Mais, dans la perspective le l'Europe de 92, quelle est la ville française qui n'a pas envie de renforcer son pouvoir d'attraction et son rayonnement touristique? Ce qui fait que l'objectif du "Il fait bon vive dans notre villet" va concerner a la fois les populations autochtones et tous les visiteurs que l'on souhaite attirer. Dans cette optique, la mise en valeur du patrimoine sous toutes ses formes (historique, culturelle, architecturale, nationale, scientifique ou technique...) n'est plus suffisante. Tout ce qui concerne l'offre tourisme-loisirs, les hébergements comme l'accueil et l'animation, va egalement faire partie de l'image que l'on souhaite donner à la ville pour la valoriser. Même les quartiers les plus défavorisés ne pourront pas être ignorés. On ne cherchera plus à les eacher mais à les réhabiliter et à offrir à leurs habitants de meilleurs services. Nous parlons ici, non seulement des coeurs anciens des villes françaises qui constituent souvent un atout de première grandeur pour le tourisme urbain mais aussi des quartiers périphériques. Des animations sportives ou culturelles réalisées à partir d'equipements structurants sont le moyen de multiplier les echanges entre populations.

Un parc urbain tourné, par exemple, vers la sauvegarde d'espèces végétales, peut avoir une fonction touristique. Tout comme la visite d'un ancien site industriel aménagé.

En conclusion, on peut s'attendre à ce que les loisirs de courte duree des Européens se développent fortement dans les annés à venir. Les "visites urbaines" ne vont pas concerner seulement les capitales mais aussi toutes les métropoles qui auront su se créer une image originale et attractive. Plusieurs villes françaises qui, au départ, n'étaient pas purement touristiques, ont dès à présent pris cette orientation. Citons, à titre d'exemple La Rochelle, Montpellier, Toulouse ou Rennes. Mais ceci peut faire I'objet d'un autre article, tout comme le problème des loisirs en milieu rural. 